\title{
Erratum to: The Auditory Enhancement Effect is Not Reflected in the $80-\mathrm{Hz}$ Auditory Steady-State Response
}

\author{
Samuele Carcagno, ${ }^{1,2}$ Christopher J. Plack, ${ }^{2,3}$ Arthur Portron, ${ }^{1}$ Catherine Semal, ${ }^{1}$ \\ AND Laurent Demany ${ }^{1}$ \\ ${ }^{1}$ Institut de Neurosciences Cognitives et Intégratives d'Aquitaine, Université de Bordeaux and CNRS, Bordeaux, France \\ ${ }^{2}$ Department of Psychology, Lancaster University, Lancaster, LA1 4YF, UK \\ ${ }^{3}$ School of Psychological Sciences, University of Manchester, Manchester Academic Health Science Centre, Manchester, M13 9PL, \\ $U K$
}

Erratum to: JARO

$$
\text { DOI } 10.1007 / \text { s10162-014-0455-y }
$$

The $y$-axis scale in Fig. 3 was incorrectly shifted upwards because of a coding error, and the unit of measure in the y-axis label was incorrectly reported as $\mu V$ instead of $\mu V^{2}$. The correct figure is shown below.

A

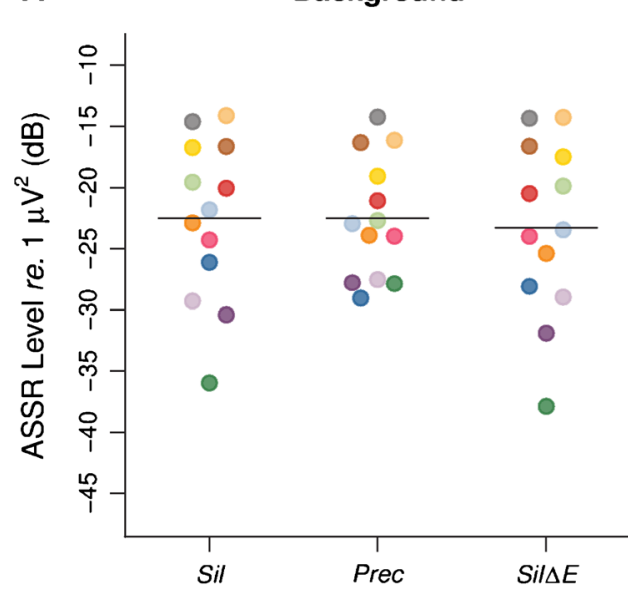

B

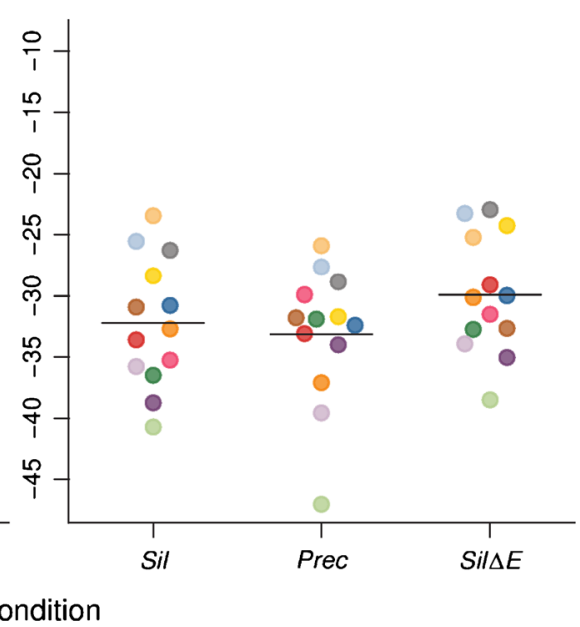

FIG. 3. A ASSR levels for the background components as a function of precursor type. B ASSR levels for the target component as a function of precursor type. Each colored point represents an individual listener. The color code is the same as in Figure 2. The horizontal segments represent the across-listener averages

The online version of the original article can be found at http:// dx.doi.org/10.1007/s10162-014-0455-y. 